\title{
植物糖基转移酶的结构与机理及糖基化工程的 研究进展
}

刘美手 ${ }^{1,2^{*}}$, 王丹丹 ${ }^{1,2}$, 秦超 ${ }^{3^{*}}$, 王小强 ${ }^{1,2^{*}}$, 沈月全 ${ }^{1,4}$

1. 南开大学药物化学生物学国家重点实验室, 天津 300350 ;

2. 南开大学药学院, 天津 300350 ;

3. 天津医科大学总医院麻醉科, 天津 300052 ;

4. 南开大学生命科学学院, 天津 300071

*联系人, E-mail: liumeizi503@163.com; qinchao0828@126.com; xq wang001@yahoo.com

收稿日期：2019-08-13; 接受日期：2019-08-27; 网络版发表日期：2019-09-06

国家重点研发计划(批准号: 2017YFA0504801, 2018YFA0901800)、天津市自然科学基金(批准号: 15JCZDJC65500)和南开大学交叉融合科学 基金(批准号: 63191138)资助

摘要 糖基转移酶(glycosyltransferases, GTs)广泛存在于各种有机体中, 通过糖基化反应参与维持细胞代谢稳态. 糖基转移酶能够识别多种受体，催化活化的糖基从供体分子转移到受体分子上，改变受体分子的化学稳定性、水 溶性以及受体分子的转运能力和生物活性等，进而有助于提高其生物利用度和生物活性等。许多被糖基化修饰的 化合物成为药物分子的重要来源. 然而, 天然产物中的糖苷类化合物存在含量低、提取难度大和提取产物纯度差 等问题. 在利用化学合成方法合成糖苷类化合物的过程中, 无法实现特定位点的糖基化修饰, 同时原料试剂和副产 物易对环境造成污染. 因此, 近年来对糖基转移酶的研究日渐增多. 本文简要综述了植物糖基转移酶的结构和生物 技术应用的研究进展, 为基于植物糖基转移酶结构的糖基化工程和生物活性糖苷化合物的生产提供有用信息.

关键词 植物糖基转移酶, 糖基化修饰, 晶体结构, 生物技术应用

天然产物具有结构新颖性和多样性的特点，同时 具有多种生物活性, 是新药开发研究的重要来源之

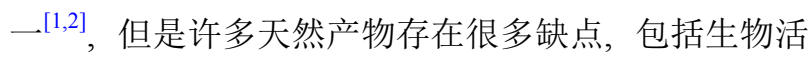
性较差、生物利用度低、发挥作用的特异性差以及毒 副作用大等，因此需要通过结构修饰和改造来改善天 然产物的性质 ${ }^{[3]}$. 天然产物的结构修饰和改造包括乙 酰化、甲基化、糖基化、羟基化等，这些修饰可丰富 天然产物的多样性，同时可以不同程度地提高天然产
物的生物活性和生物利用度, 其中糖基化修饰在改善 溶解度和提高稳定性等方面的效果显著 ${ }^{[4 \sim 6]}$. 然而天然 产物中的糖苷类化合物存在含量低、提取难度大和提 取产物纯度差等问题，在用化学合成的方法合成糖苷 类化合物的过程中, 无法实现特定位点的糖基化修饰. 同时, 原料试剂和副产物易对环境造成污染. 因此, 近 年来对利用生物合成的方法合成糖苷类化合物的研究 日渐增多. 本文简要综述植物糖基转移酶(glycosyl-

引用格式: 刘美子, 王丹丹, 秦超, 等. 植物糖基转移酶的结构与机理及糖基化工程的研究进展. 中国科学: 生命科学, 2019, 49: 1133-1142 Liu M Z, Wang D D, Qin C, et al. Research progress in understanding the structure, mechanism, and engineering of plant glycosyltransferases (in Chinese). Sci Sin Vitae, 2019, 49: 1133-1142, doi: 10.1360/SSV-2019-0164 
transferases，GTs)的结构、催化机制以及生物技术应 用的研究进展, 为基于植物糖基转移酶结构的糖基化 工程发展和具有生物活性糖苷化合物的工业化生产奠 定基础.

糖基转移酶 $\mathrm{GTs}$ 是高度分化的超级酶家族, 糖基 转移酶的糖基受体底物多种多样, 不仅包括单糖、葟 糖和多糖，还包括各种非碳水化合物，例如蛋白质、 抗生素和脂质、类固醇以及小分子天然产物等 ${ }^{[7]}$. 糖 基供体分子主要包括常见的糖类、核苷-2-磷酸糖、 UDP(uridine diphosphate)-葡萄糖、UDP-葡萄糖醛 酸、UDP-鼠李糖和UDP-木糖等 ${ }^{[8 \sim 10]}$.

\section{1 糖基转移酶的分类}

随着对糖基转移酶研究的深入, 越来越多的 GTs 被鉴定出来, 截至目前, 碳水化合物活性酶数据库 CAZy(carbohydrate-active enzyme database)中共收录 了高达550978 个不同生物物种 $\mathrm{GT}$ 的氨基酸序列 (http://www.cazy.org/GlycosylTransferases.html), 包括 11654 个尚未分类的序列. 根据GT序列的相似度、催 化底物的特异性和催化产物的立体化学结构, CAZy 数据库将糖基转移酶分为 107 个家族(GT1 GT107) ${ }^{[11]}$, 其中 $\mathrm{GT} 1$ 是含糖基转移酶数目最多的家族.

根据受体分子的糖基化位点分类, 糖基转移酶催 化形成 $\mathrm{O}-\mathrm{C}$ 糖苷键、 N-C糖苷键、C $-\mathrm{C}$ 糖苷键和 S-C 糖苷键, 相应的糖基转移酶分别是O-GT, N-GT, C-GT 和 S-GT, 目前研究较多的植物糖基转移酶大多为 $\mathrm{O}-\mathrm{GT}$, 例如教㢣苜宿 (Medicago truncatula) $\mathrm{UGT} 71 \mathrm{G} 1^{[12]}$ 、酿酒葡萄(Vitis vinifera) $\mathrm{VvGT} 1^{[13]}$ 、拟 南芥 (Arabidopsis thaliana) $\mathrm{UGT} 89 \mathrm{C} 1^{[10]}$ 、教䓠苜宿 UGT $85 \mathrm{H} 2^{[14]}$ 等O-GT的三维结构和催化机制已得到深 入研究. 此外, 拟南芥UGT76C1和UGT76C2 能够催化 $\mathrm{N}-\mathrm{C}$ 糖苷键生成, 属于N-GT ${ }^{[15]}$. 而菾麦 (Fagopyrun esculentum)UGT708C1/UGT708C2则属于C-GT, 它能够 催化2-羟基黄酮类化合物产生 C-C糖苷键 ${ }^{[16]}$. 另外, 具 有双重催化功能的糖基转移酶的基因逐渐被鉴定出 来, 例如水稻(Oryza sativa)OsCGT可以催化形成O-C 糖苷键和C-C糖苷键 ${ }^{[17]}$, 玉米(Zea mays)UGT708A6对 柚皮素表现出 $\mathrm{O}$-糖基化的催化活性, 而对2-羟基柚皮 素则表现出C-糖基化的催化活性 ${ }^{[18]}$. 拟南芥UGT72B1 具有催化 $N-C$ 糖苷键和 $O-C$ 糖苷键形成的活性 ${ }^{[19]}$, 苏
云金杆菌(Bacillus thuringiensis)ThuS可以同时催化 O-C 糖苷键和 S-C糖苷键的形成, 且 ThuS 催化的 S-糖 基化比 $\mathrm{O}$-糖基化更有效 ${ }^{[20]}$, 土壤细菌弗氏链霉菌 (Streptomyces fradiae Tü2717)UrdGT2具有催化C-C糖 苷键生成的活性, 同时也可以催化O-C糖苷键生成 ${ }^{[21]}$. 具有多重催化功能的糖基转移酶也被鉴定出来, 例如, 2008年报道了抗生素链需菌(Streptomyces antibioticus) 中的一种糖基转移酶 $\mathrm{OleD}$, 这是第一个可以催化 $\mathrm{O}-\mathrm{C}$, S-C 和N-C糖苷键生成的糖基转移酶 ${ }^{[22]}$. 2014年报道 的从红花(Carthamus tinctorius) 中鉴定出来的新型糖 基转移酶UGT73AE1可以识别与接受许多结构不同的 受体, 催化形成O-C, S-C 和 $-C$ C糖苷键的同时, 还具有 催化其逆反应的活性 ${ }^{[23]}$. 本实验室 ${ }^{[24]}$ 最近鉴定的青钱 柳(Cyclocarya paliurus)糖基转移酶CpGT1也可以催化 $\mathrm{O}-\mathrm{C}, \mathrm{S}-\mathrm{C}$ 和N-C糖苷键的生成.

根据糖基转移酶的三维结构的折叠特征, 可以将 糖基转移酶分为 GT-A, GT-B, GT-C和 GT-D4种 (图1) ${ }^{[25]}$. 其中 GT-A 是核苷二磷酸转移酶超家族(包括 核苷酸转移酶), 其特征是含有保守的DXD(Asp-XaaAsp)基序，用于结合GT-A超家族成员催化反应必需的 二价金属离子 $\left(\right.$ 多数为 $\left.\mathrm{Mn}^{2+}\right)$. GT-A类蛋白空间折叠的 特征是含有两个排列紧密的 $\beta / \alpha / \beta$ 结构域, 其 $\mathrm{N}$ 端结构 域参与核苷酸糖供体的识别, 而 $\mathrm{C}$ 端结构域主要与受 体底物相互作用 ${ }^{[26]}$. 这种由生物学家Rossmann等人 ${ }^{[27]}$ 发现的 $\beta / \alpha / \beta$ 结构域被称为Rossmann折叠(Rossmannfolds). 1999年, Charnock和Davies ${ }^{[28]}$ 解析了首个GT-A 类折叠的核苷酸二磷酸糖转移酶SpsA(Bacillus subti$l i s$ )的结构(PDB 1QGQ). 除了糖基转移酶之外, GT-B 超家族成员还包括参与糖代谢的其他酶, 如糖差向异 构酶(UDP-GlcNAc 2-epimerase) ${ }^{[29]}$. GT-B类折叠同样 是由两个Rossmann折叠的 $\beta / \alpha / \beta$ 结构域构成, 不同的是 其 $\mathrm{N}$ 端结构域参与结合糖基受体, 而 $\mathrm{C}$ 端结构域则参与 结合糖基供体, 它们之间的连接不太紧密, 两个结构域 “相互面对”，配体的结合与相对取向的构象变化有 关 ${ }^{[30]}$. GT-B类折叠的C端结构域具有高度保守的PSPG (putative secondary plant glycosyltransferase)结构模体, 该结构域在糖基供体识别和催化过程中发挥重要的作 用. 另外, 与GT-A超家族不同, GT-B超家族的成员催化 作用不依赖于二价金属离子 ${ }^{[31]}$, 尚无证据证明其催化 活性与金属离子结合之间的关联. 1999年, Gastinel等 人 $^{[32]}$ 从牛血清中分离并解析了首个 GT-B类折叠的 
A

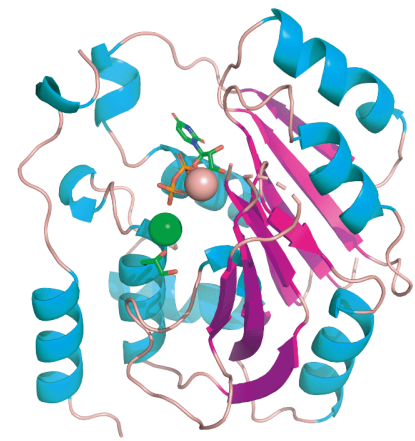

C

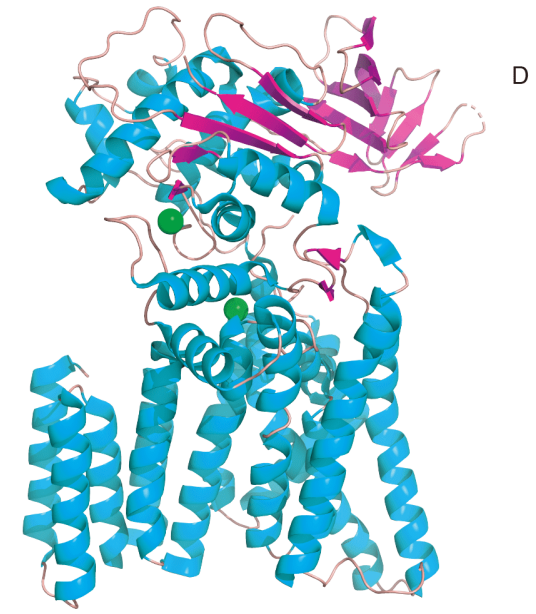

B
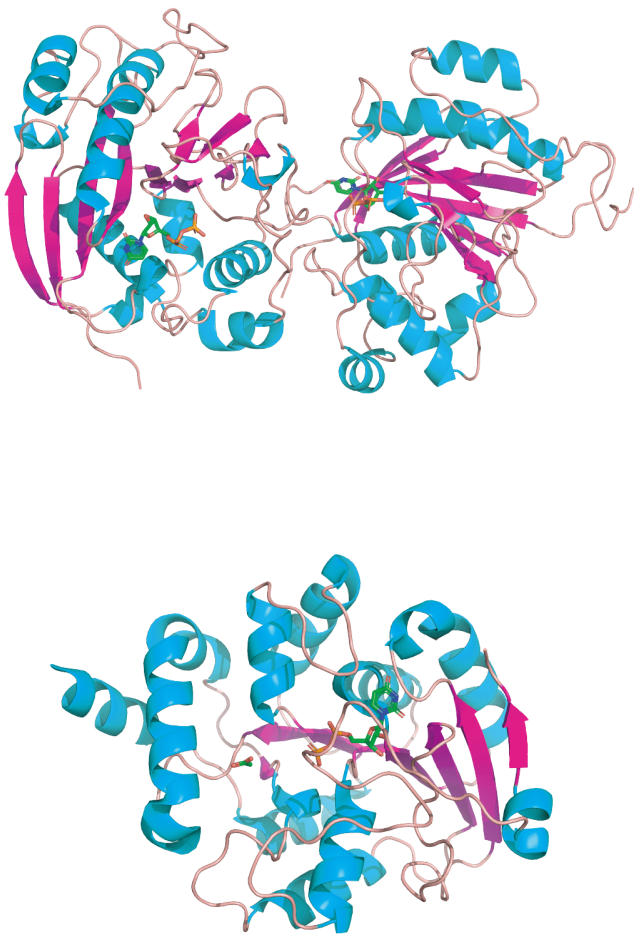

图 1 糖基转移酶四种不同的折叠方式. A: GT-A：枯草芽孢杆菌(Bacillus subtilis)核苷酸二磷酸糖转移酶SpsA的结构(PDB 1QGQ); B: GT-B: $\beta 1,4-$ 半乳糖基转移酶 $\beta 4 G a l-T 1(B o v i n e)$ 的结构(PDB 1FR8); C: GT-C: 红嘴鸥弯曲杆菌寡糖基转移酶PglB的全 长的晶体结构(PDB 3RCE); D: GT-D: 副血链球菌未被表征的“功能未知”的结构域DUF1792的结构(PDB 4PFX)

Figure 1 Four different folding patterns of glycosyltransferases (GTs). A: GT-A: the structure of nucleotide-diphospho-sugar transferase spsA from Bacillus subtilis (PDB 1QGQ). B: GT-B: the structure of bovine $\beta 1$,4-galactosyltransferase T1 (PDB 1FR8). C: GT-C: the structure of full-length oligosaccharyltransferase PglB from Campylobacter lari (PDB 3RCE). D: GT-D: the structure of domain of unknown function 1792 (DUF1792) from Streptococcus parasanguinis (PDB 4PFX)

$\beta 1,4$-半乳糖基转移酶 $\beta 4 \mathrm{Gal}-\mathrm{T} 1$ 的晶体结构(PDB 1FR8)。GT-C超家族由位于内质网或质膜上较大的疏 水蛋白组成，包括 $\mathrm{N}$ 端的跨膜区和C端的环状区域，在 第一个胞外环中具有被修饰的DXD基序，目前没有证 据证明GT-C超家族的DXD基序与GT-A的DXD基序有 共同的进化起源 ${ }^{[25]}$. 2011年, Lizak等人 ${ }^{[33]}$ 解析了第一 个 $\mathrm{GT}-\mathrm{C}$ 超家族的寡糖基转移酶PglB 的全长的晶体结 构(PDB 3RCE), 该结构显示跨膜区对于肽的结合和催 化都是不可或缺的. 2014年, Zhang等人 ${ }^{[34]}$ 以 $1.4 \AA$ 分辨 率解析了副血链球菌(Streptococcus parasanguinis) dGT1的未被表征的“功能未知”的结构域DUF1792的 晶体结构(PDB 4PFX). 该结构域采用一种全新的折叠 方式, 并具有葡萄糖基转移酶的活性, 该结构域的催化
活性依赖于二价金属离子，最终被命名为GT-D类糖基 转移酶折叠. 由于该结构域在细菌中高度保守, 在真核 生物中不存在, 因此可以作为新的抗菌靶标进行深入 研究.

\section{2 糖基转移酶的催化机制}

根据糖基化底物和产物的立体化学异构性，糖基 转移酶的催化机制可以分为两类, 即构型保持型(retention)和构型翻转型(inversion) ${ }^{[26]}$ (图2). 其中构型翻 转型的催化过程是受体分子的活泼氢被糖基转移酶的 碱性氨基酸夺取, 随后受体分子上的氧负离子从另外 一侧进攻供体分子的异头碳，形成一个oxocarbenium 
A

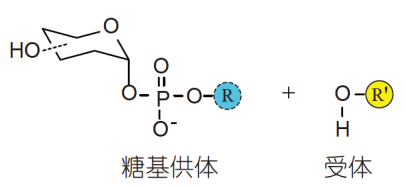

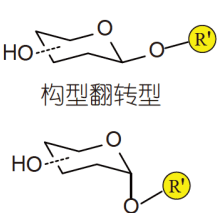

构型保持型
B

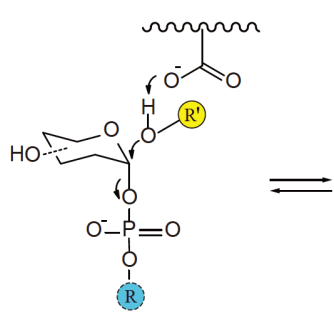

C

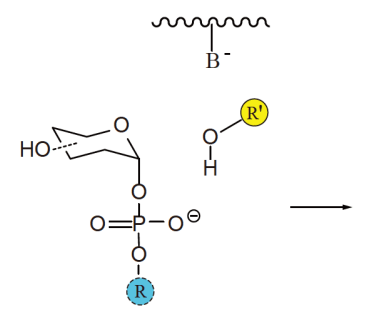

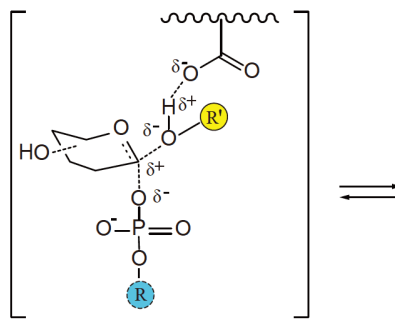

oxocarbenium离子样

过渡态

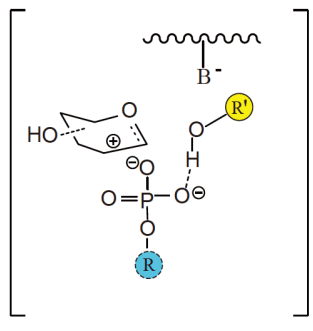

短暂的oxocarbenium样过渡态

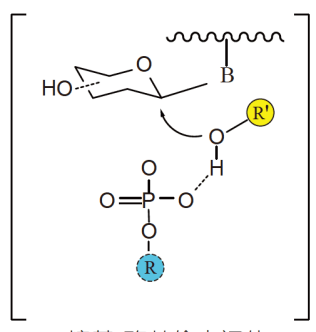

糖基-酶共价中间体

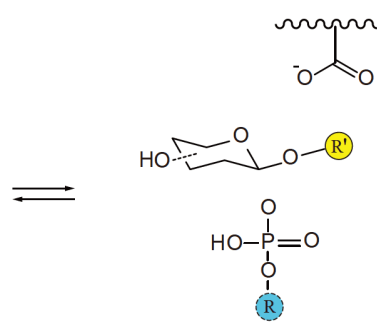

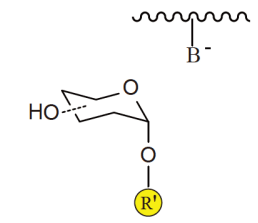<smiles>[13CH3]P(=O)([O-])[18O]</smiles>

图 2 糖基转移酶的催化机制. A: 相对于糖基供体底物的构型, 糖基转移酶催化糖单体转移的方式包括构型翻转型和构型保 持型; B: 糖基转移酶构型翻转型的催化机理; C: 糖基转移酶构型保持型的催化机制

Figure 2 The catalytic mechanisms of glycosyltransferases (GTs). A: GTs catalyze the transfer of sugars by either "inversion" or "retention"of the conformation with respect to the sugar donor substrates. B: the catalytic mechanism of "inversion" GTs. C: the catalytic mechanisms of "retention" GTs

离子样过渡态，磷酸基团脱离，完成构型翻转催化过 程 ${ }^{[35]}$ ，该催化过程类似于翻转型的糖苷水解酶(glycoside hydrolases, GHs)催化糖苷键断裂的过程 ${ }^{[36]}$. 而构 型保持型的催化机理则存在很大争议 ${ }^{[26]}$, 首先提出的 是形成糖基-酶共价中间体的双置换机制，糖基转移酶 活性中心的碱性氨基酸从另一侧对受体分子的异头碳 进行亲核进攻，磷酸基团脱离，形成糖基-酶共价中间
体，随后受体分子的活泼氢与磷酸基团的氧原子形成 氢键，此时的氧原子从另一侧对受体分子的异头碳进 行亲核进攻, 完成构型保持的催化过程 ${ }^{[35]}$. 为了支持 这一催化模型, Monegal和Planas ${ }^{[37]}$ 报道了通过叠氮化 钠对 $\alpha 3$-半乳糖基转移酶 $(\alpha 3 \mathrm{GalT})$ 的突变形式的化学 补救, 这种化学补救的产物是糖叠氮化物的翻转形式, 这与双置换机制的第一步一致. 然而在缺乏其他可行 
的共价中间体的明确实验证据的情况下, 另一种催化 机制SNi“内部返回”(internal return)被提出, 该机理提 出亲核进攻和离去基团的脱离发生在糖基的同侧, 形 成一个短暂的oxocarbenium样过渡态, 先后发生受体 $\mathrm{C}-\mathrm{O}$ 糖苷键的形成和糖基与磷酸基团之间 $\mathrm{C}-\mathrm{O}$ 键的断 裂, 最终完成构型保持的催化过程 ${ }^{[38-40]}$. 总之, 构型保 持型的糖基转移酶是否可以通过不同的催化机制进行 需要进一步实验证明.

\section{3 植物糖基转移酶结构研究现状}

植物糖基转移酶的种类众多, 催化底物广泛, 糖基 化可直接影响化合物水溶性、稳定性和生物活性 等 ${ }^{[41 \sim 45]}$. 此外, 糖基转移酶对天然产物进行糖基化修 饰可得到新的生物活性化合物, 对于现代药物研发具 有重要的意义. 随着新的糖基转移酶不断被鉴定出来, 同时糖基转移酶的三维结构不断被解析, 其三维结构 揭示了酶与底物相互作用的细节和催化机制, 为进一 步阐释底物特异性和专一性提供了依据, 有助于糖基 转移酶在生物催化、基因工程等方面的应用.

目前解析的植物糖基转移酶的结构有 11 个，包括 模式生物拟南芥中的UGT72B $1^{[19]}, \mathrm{UGT} 74 \mathrm{~F} 2^{[46]}$, $\mathrm{UGT} 89 \mathrm{C} 1^{[10]}$; 跧藜苜宿中的 $\mathrm{UGT} 71 \mathrm{G} 1^{[12]}$, $\mathrm{UGT} 78 \mathrm{G1} 1^{[47]}$, UGT85H2 $2^{[14]}$; 酿酒葡萄中的 $\mathrm{VvGT1}^{[13]}$; 蝶豆 (Clitoria ternatea) 中的UGT78 $\mathrm{K}^{[48]}$; 水稻中的 Os79 ${ }^{[49,50]}$; 蓼蓝(Polygonum tinctorium)中的PtUGT1 ${ }^{[51]}$; 甜叶菊(Stevia rebaudiana)中的UGT76G1 ${ }^{[52,53]}$. 具体的 三维结构信息汇总见表1.

目前已报道的这些植物糖基转移酶的结构均属于 GT-B折叠方式, 含有两个Rossmann折叠的 $\beta / \alpha / \beta$ 结构 域, $\mathrm{N}$ 端结构域和 $\mathrm{C}$ 端结构域之间形成一条细长的缝 隙, 其中 $\mathrm{N}$ 端结构域参与结合糖基受体, 因植物糖基转 移酶受体种类的多样性, N端结构域的保守性较差, 但 是参与糖基化催化反应的重要氨基酸位点(His/Asp)是 高度保守的, 而糖基供体则结合在C端结构域相对保 守的PSPG区域.

\section{1 植物糖基转移酶的供体结合区域}

糖基供体通过与植物糖基转移酶 $\mathrm{C}$ 端结构域的 PSPG区域的氨基酸残基之间的多个氢键相互作用, 稳 定地结合在糖基转移酶的PSPG区域, 其中PSPG区域
的最后两个氨基酸残基(如MtUGT71G1中的Glu381和 Gln382, VvGT1中的Asp374和Gln375)被认为是糖基识 别的关键氨基酸 ${ }^{[12,13]} .2019$ 年, 本课题组 ${ }^{[10]}$ 报道了关于 鼠李糖转移酶AtUGT 89 C 1 的结构及功能研究, 发现 Asp356突变为Ala后, AtUGT89C1对鼠李糖的转移活 性完全丧失，而 $\mathrm{H} 357 \mathrm{Q}$ 突变体则表现出对鼠李糖和葡 萄糖的双重转移活性，进一步证明了PSPG区域最后 两个氨基酸残基在糖基识别过程中的关键作用. 此外, 位于糖基供体结合中心的相对保守的苏氨酸 (MtUGT71G1中的Thr143, VvGT1中的Thr141) 被认为 在糖基供体识别过程中发挥非常重要的作用, VvGT1 中的Thr141突变为Ala之后活性一定程度的丧失也证 明了这一猜想 ${ }^{[13]}$,AtUGT89C1中的Pro147和Ile148与 鼠李糖环6-位甲基形成疏水相互作用，对鼠李糖基的 识别非常重要 ${ }^{[10]}$.

\section{2 植物糖基转移酶的受体结合区域}

植物糖基转移酶的 $\mathrm{N}$ 端结构域负责结合糖基受体, 由于 $\mathrm{N}$ 端结构域之间存在较大的差异性, 导致不同糖 基转移酶的糖基受体种类具有多样性。例如， MtUGT71G1 识别三萜类和黄酮类化合物作为糖基受 体, 主要在黄酮类化合物的 $3^{\prime}-\mathrm{O}$ 生成葡萄糖基化产物, 而双点突变Y202A/F148V使得MtUGT71G1对解皮素 的专一性发生改变, 使糖基化位点由 $3^{\prime}-\mathrm{OH}$ 变成 $3-\mathrm{OH}^{[12]} ; \mathrm{VvGT} 1$ 对许多不同的黄酮类化合物(包括檞 皮素和山奈酚)具有催化活性，生成3-O葡萄糖苷 ${ }^{[13]}$; MtUGT85H2可以识别黄酮和异黄酮类化合物作为糖 基受体, 但是具有一定的立体选择性, 例如当以鹰嘴豆 芽素 $\mathrm{A}$ 和染料木黄酮为糖基受体时, 主要生成7-O葡萄 糖苷, 而以山奈酚和檞皮素作为底物时, 主要生成3-O葡萄糖苷. 此外, 该酶对底物的活性测试和动力学分析 结果显示, MtUGT85H2对黄酩醇的特异性明显高于异 黄酮类化合物. 不同的糖基转移酶 $\mathrm{N}$ 端结构域又具有 一定的相似性, 多个疏水性氨基酸组成疏水环境, 糖 基受体则主要结合在这个疏水区域. 例如，在 VvGT1 与山奈酚的复合物结构中, 山奈酚结合在由Phe 15 , Ile87, Phe121, Phe200, Val281和Phe372等组成的疏水 口袋里 ${ }^{[13]}$; 在AtUGT72B1与 与CP $(2,4,5$-三氯苯酚 $)$ 的复 合物结构中, TCP主要通过疏水相互作用同Leu118, Phe119，Phe148，Leu183和Leu197组成的疏水口袋结 合; 在AtUGT $89 \mathrm{C} 1$ 与葪皮素的复合物结构中，槲皮素 
表 1 植物糖基转移酶的晶体结构信息

Table 1 Crystal structure information of plant glycosyltransferases

\begin{tabular}{|c|c|c|c|c|}
\hline UGT 名称 & 分辨率 $(\AA)$ & 晶体复合物 & PDB编号 & 发表年份 \\
\hline \multirow{2}{*}{ MtUGT71G1 } & 2.0 & UDP & $2 \mathrm{ACV}$ & \multirow{2}{*}{2005} \\
\hline & 2.6 & UDP-glucose & $2 \mathrm{ACW}$ & \\
\hline \multirow{3}{*}{ VvGT1 } & 1.9 & UDP & $2 \mathrm{C} 1 \mathrm{X}$ & \multirow{3}{*}{2006} \\
\hline & 1.9 & UDP-2FGlc, kaempferol & $2 \mathrm{C} 1 \mathrm{Z}$ & \\
\hline & 2.1 & UDP and quercetin & $2 \mathrm{C} 9 \mathrm{Z}$ & \\
\hline MtUGT85H2 & 2.1 & Apo & 2PQ6 & 2007 \\
\hline \multirow{3}{*}{ AtUGT72B1 } & 1.45 & UDP & $2 \mathrm{VCH}$ & \multirow{3}{*}{2007} \\
\hline & 1.75 & UDP and Tris & 2VG8 & \\
\hline & 1.9 & UDP-2FGlc, 2,4,5-trichlorophenol & $2 \mathrm{VCE}$ & \\
\hline \multirow{2}{*}{ MtUGT78G1 } & 2.1 & UDP & $3 \mathrm{HBJ}$ & \multirow{2}{*}{2009} \\
\hline & 2.1 & UDP and myricetin & $3 \mathrm{HBF}$ & \\
\hline \multirow{5}{*}{ CtUGT78K6 } & 1.85 & Apo & $3 \mathrm{WC} 4$ & \multirow{5}{*}{2015} \\
\hline & 1.85 & UDP & 4WHM & \\
\hline & 2.55 & Delphinidin & 4REM & \\
\hline & 2.7 & Petunidin & 4REN & \\
\hline & 1.75 & Kaempferol & 4REL & \\
\hline \multirow{3}{*}{ Os79 } & 1.8 & UDP-open & $5 \mathrm{TME}$ & \multirow{3}{*}{2016} \\
\hline & 2.3 & UDP-closed & $5 \mathrm{TMB}$ & \\
\hline & 2.4 & UDP-2FGlc, trichothecene & 5TMD & \\
\hline Os79Q202A & 1.47 & UDP & $6 \mathrm{BKO}$ & \multirow{4}{*}{2017} \\
\hline Os79H122A/L123A & 1.29 & UDP & $6 \mathrm{BK} 1$ & \\
\hline Os79T291V & 1.58 & UDP & $6 \mathrm{BK} 2$ & \\
\hline Os79 & 2.17 & UDP, deoxynivalenol-3-glucose & 6BK3 & \\
\hline \multirow{2}{*}{ AtUGT74F2 } & 2.56 & UDP, salicylic acid (BA) & $5 \mathrm{U} 6 \mathrm{M}$ & \multirow{5}{*}{2017} \\
\hline & 2.0 & UDP, 2-bromobenzoic acid (2BA) & $5 \mathrm{U} 6 \mathrm{~S}$ & \\
\hline AtUGT74F2T15S & 2.0 & UDP, salicylic acid & $5 \mathrm{U} 6 \mathrm{~N}$ & \\
\hline AtUGT74F2T15A & 2.0 & UDP, 2-bromobenzoic acid & $5 \mathrm{~V} 2 \mathrm{~K}$ & \\
\hline AtUGT74F2T15S & 1.8 & UDP, 2-bromobenzoic acid & $5 \mathrm{~V} 2 \mathrm{~J}$ & \\
\hline PtUGT1 & 2.14 & Indoxyl sulfate & $5 \mathrm{NLM}$ & 2018 \\
\hline \multirow{4}{*}{ AtUGT89C1 } & 2.7 & Apo & $6 \mathrm{IJ} 7$ & \multirow{4}{*}{2019} \\
\hline & 3.0 & UDP & 6IJ9 & \\
\hline & 3.21 & UDP- $\beta$-L-rhamnose & 6IJA & \\
\hline & 3.2 & Quercetin & 6IJD & \\
\hline \multirow{3}{*}{ UGT76G1 } & 1.8 & (SeMet) UDP & 6086 & \multirow{3}{*}{2019} \\
\hline & 1.75 & UDP & 6087 & \\
\hline & 1.99 & UDP, rebaudioside A & 6088 & \\
\hline UGT76G1 & 1.69 & UDP & $6 \mathrm{INF}$ & \multirow{4}{*}{2019} \\
\hline UGT76G1H25A & 1.7 & UDP, GOL & $6 \mathrm{ING}$ & \\
\hline UGT76G1 & 2.1 & AQ9, GOL, UDP & $6 \mathrm{INH}$ & \\
\hline UGT76G1 & 1.7 & AQ9, AUO, GOL, UDP & $6 \mathrm{INI}$ & \\
\hline
\end{tabular}


结合在Phe16, Phe95, Ile91, Leu88, Phe165, Ala355, Phe 359 等组成的疏水口袋里 ${ }^{[10]}$. 因此糖基受体与植物 糖基转移酶的 $\mathrm{N}$ 端结构域之间主要通过氢键和疏水作 用实现相互作用，此外有些蛋白质分子内的相互作用 也会间接地影响植物糖基转移酶的活性 ${ }^{[14]}$.

\section{4 植物糖基转移酶的生物应用及展望}

随着对植物糖基转移酶的结构和生物活性糖苗化 合物的深入研究，更多的植物糖基转移酶被应用在代 谢工程和基因工程. 人参皇苷 Rh2 和 Rg3 具有抗癌活性 和低毒性，二者是用于癌症预防和治疗的潜在候选 物 ${ }^{[54 \sim 57]}$, 然而人参中的生物活性成分三萜皇苷 Rh2 和 $\mathrm{Rg} 3$ 的浓度极低. 人参中含有两种葡萄糖基转移酶 UGTPg29和UGTPg45, 其中UGTPg45选择性地将葡萄 糖部分转移到原人参二醇(protopanaxadiol)及其人参 㿝苷的C3 位羟基上, UGTPg29可选择性地将葡萄糖部 分转移到Rh2的C3 葡萄糖上，形成1-2糖苷键. 2015年, Wang等人 ${ }^{[58]}$ 构建了含有这两个糖基转移酶的酵母工 程菌，通过微生物发酵利用单糖生产 Rh2或 Rg3，突破 了从人参植物中提取人参皇苷的传统方法，提供了一 种高效简捷的人参皇苷生物合成途径.

乙酰水杨酸(阿司匹林)是一种非阿片类和非甾体 类抗炎药物，是应用最早和最广泛的解热镇痛药和抗 风湿药. 商业化生产的阿司匹林的前体是水杨酸，其 主要来源是石油，这导致了可持续性发展的问题. 2016年，Ahmadi等人 ${ }^{[59]}$ 使用大肠杆菌(Escherichia coli)作为异源宿主，构建含有拟南芥糖基转移酶 UGT74F1的表达体系，完成了水杨酸2-O- $\beta-\mathrm{D}$-葡萄糖 苷(salicylic acid 2-O- $\beta$-D-glucose, SAG)的生物合成, 应用代谢工程并改变翻译起始速率以优化水杨酸 SA
和SAG的生产工艺. 然后利用共培养方法进一步促进 SA向 SAG 的完全转化. SAG 的生物活性评估证实, RAW264.7巨噬细胞的NO和ROS(reactive oxygen species)的抗炎特性与阿司匹林相当或更好, 同时对细胞 生存能力没有不利影响. 最终合成了阿司匹林的活性 类似物SAG, 同时提供了SAG的生物合成途径.

肉桂醇糖苷(cinnamic alcohol glycosides, CAGs) 是 珍贵药用植物红景天(Rhodiola rosea) 的关键活性成分, 具有多种药理活性. 肉桂醇二糖(Rosavin)和红景天苷 的含量是衡量红景天提取物质量的关键标准. Rosavin 和类似物的供应受到化学合成方法的低效率和自然资 源短缺的限制. 2019年, $\mathrm{Bi}$ 等人 ${ }^{[60]}$ 通过基因工程改造大 肠杆菌菌株实现了一系列Rosavin类似物的从头合成. 其中有一个环节用来自拟南芥的UGT73C5 和来自长 春花 (Catharanthus roseus) 的糖链延长糖基转移酶 CaUGT3依次催化形成非天然肉桂醇二葡萄糖苷，经 过后续反应，获得7种Rosavin类似物，包括6种非天然 化合物. 这项工作丰富了CAG的结构多样性, 为大规 模发酵生产Rosavin类似物奠定了重要基础.

植物糖基转移酶在改善天然产物成药性，提高现 有药物的生物活性和生物利用度, 促进新药的研发等 方面具有很大的应用潜力. 此外, 植物糖基转移酶在 改善植物对内外毒素的抗性、调节植物体内激素平衡 等方面也具有巨大应用前景. 但是植物糖基转移酶在 应用过程中存在一些问题，例如由于其催化底物专一 性的特点, 导致糖基化产物多样性受限; 糖基供体的 高成本限制了糖苷产物的大规模合成等. 总的来说, 随着对植物糖基转移酶结构和功能以及催化机制的研 究不断深入, 结合基因工程和代谢工程的技术手段, 植 物糖基转移酶必将在糖苷化合物及药物的工业生产和 新药研发等领域得到广泛应用.

\section{参考文献}

1 Li R. Natural product-based drug discovery. Med Res Rev, 2016, 36: 3

2 Katz L, Baltz R H. Natural product discovery: Past, present, and future. J Ind Microbiol Biotechnol, 2016, 43: 155-176

3 Yao H, Liu J, Xu S, et al. The structural modification of natural products for novel drug discovery. Expert Opin Drug Discov, 2017, 12: 121-140

4 Muñoz-Antoli C, Cortés A, Sotillo J, et al. Differential expression and glycosylation of proteins in the rat ileal epithelium in response to Echinostoma caproni infection. J Proteom, 2014, 101: 169-178

5 Liang D M, Liu J H, Wu H, et al. Glycosyltransferases: Mechanisms and applications in natural product development. Chem Soc Rev, 2015, 44: $8350-8374$

6 Ilmberger N, Rabausch U. Screening glycosyltransferases for polyphenol modifications. Methods Mol Biol, 2017, 1539: 229-236 
7 Bowles D, Isayenkova J, Lim E K, et al. Glycosyltransferases: Managers of small molecules. Curr Opin Plant Biol, 2005, 8: 254-263

8 Mackenzie P I, Owens I S, Burchell B, et al. The UDP glycosyltransferase gene superfamily: Recommended nomenclature update based on evolutionary divergence. Pharmacogenetics, 1997, 7: 255-269

9 Sawada S, Suzuki H, Ichimaida F, et al. UDP-glucuronic acid: Anthocyanin glucuronosyltransferase from red daisy (Bellis perennis) flowers. Enzymology and phylogenetics of a novel glucuronosyltransferase involved in flower pigment biosynthesis. J Biol Chem, 2005, 280: 899-906

10 Zong G, Fei S, Liu X, et al. Crystal structures of rhamnosyltransferase UGT89C1 from Arabidopsis thaliana reveal the molecular basis of sugar donor specificity for UDP- $\beta$-L-rhamnose and rhamnosylation mechanism. Plant J, 2019, 68: tpj.14321

11 Lombard V, Golaconda Ramulu H, Drula E, et al. The carbohydrate-active enzymes database (CAZy) in 2013. Nucl Acids Res, 2014, 42: D490D495

12 Shao H, He X, Achnine L, et al. Crystal structures of a multifunctional triterpene/flavonoid glycosyltransferase from Medicago truncatula. Plant Cell, 2005, 17: 3141-3154

13 Offen W, Martinez-Fleites C, Yang M, et al. Structure of a flavonoid glucosyltransferase reveals the basis for plant natural product modification. EMBO J, 2006, 25: 1396-1405

14 Li L, Modolo L V, Escamilla-Trevino L L, et al. Crystal structure of Medicago truncatula UGT85H2 - insights into the structural basis of a multifunctional (iso) flavonoid glycosyltransferase. J Mol Biol, 2007, 370: 951-963

15 Hou B, Lim E K, Higgins G S, et al. N-glucosylation of cytokinins by glycosyltransferases of Arabidopsis thaliana. J Biol Chem, 2004, 279: $47822-47832$

16 Nagatomo Y, Usui S, Ito T, et al. Purification, molecular cloning and functional characterization of flavonoid $C$-glucosyltransferases from Fagopyrum esculentum M. (buckwheat) cotyledon. Plant J, 2014, 80: 437-448

17 Hao B, Caulfield J C, Hamilton M L, et al. Biosynthesis of natural and novel C-glycosylflavones utilising recombinant Oryza sativa Cglycosyltransferase (OsCGT) and Desmodium incanum root proteins. Phytochemistry, 2016, 125: 73-87

18 Falcone Ferreyra M L, Rodriguez E, Casas M I, et al. Identification of a bifunctional maize $C$ - and $O$-glucosyltransferase. J Biol Chem, 2013, 288: $31678-31688$

19 Brazier-Hicks M, Offen W A, Gershater M C, et al. Characterization and engineering of the bifunctional $N$ - and $O$-glucosyltransferase involved in xenobiotic metabolism in plants. Proc Natl Acad Sci USA, 2007, 104: 20238-20243

20 Wang H, Oman T J, Zhang R, et al. The glycosyltransferase involved in thurandacin biosynthesis catalyzes both $O$ - and $S$-glycosylation. J Am Chem Soc, 2014, 136: 84-87

21 Dürr C, Hoffmeister D, Wohlert S E, et al. The glycosyltransferase UrdGT2 catalyzes both $C$ - and $O$-glycosidic sugar transfers. Angew Chem Int Ed, 2004, 43: 2962-2965

22 Gantt R W, Goff R D, Williams G J, et al. Probing the aglycon promiscuity of an engineered glycosyltransferase. Angew Chem Int Ed, 2008, 47: $8889-8892$

23 Xie K, Chen R, Li J, et al. Exploring the catalytic promiscuity of a new glycosyltransferase from Carthamus tinctorius. Org Lett, 2014, 16: 48744877

24 Li J, Liu X, Gao Y, et al. Identification of a UDP-glucosyltransferase favouring substrate- and regio-specific biosynthesis of flavonoid glucosides in Cyclocarya paliurus. Phytochemistry, 2019, 163: 75-88

25 Liu J, Mushegian A. Three monophyletic superfamilies account for the majority of the known glycosyltransferases. Protein Sci, 2003, 12: 14181431

26 Lairson L L, Henrissat B, Davies G J, et al. Glycosyltransferases: Structures, functions, and mechanisms. Annu Rev Biochem, 2008, 77: 521-555

27 Rossmann M G, Moras D, Olsen K W. Chemical and biological evolution of a nucleotide-binding protein. Nature, 1974, 250: 194-199

28 Charnock S J, Davies G J. Cloning, crystallization and preliminary X-ray analysis of a nucleotide-diphospho-sugar transferase spsA from Bacillus subtilis. Acta Crystlogr D Biol Crystlogr, 1999, 55: 677-678

29 Wrabl J O, Grishin N V. Homology between O-linked GlcNAc transferases and proteins of the glycogen phosphorylase superfamily. J Mol Biol, 2001, 314: 365-374

30 Coutinho P M, Deleury E, Davies G J, et al. An evolving hierarchical family classification for glycosyltransferases. J Mol Biol, 2003, 328: 307317

31 Breton C, Snajdrová L, Jeanneau C, et al. Structures and mechanisms of glycosyltransferases. Glycobiology, 2006, 16: 29R-37R 
32 Gastinel L N, Cambillau C, Bourne Y. Crystal structures of the bovine $\beta$ 4galactosyltransferase catalytic domain and its complex with uridine diphosphogalactose. EMBO J, 1999, 18: 3546-3557

33 Lizak C, Gerber S, Numao S, et al. X-ray structure of a bacterial oligosaccharyltransferase. Nature, 2011, 474: 350-355

34 Zhang H, Zhu F, Yang T, et al. The highly conserved domain of unknown function 1792 has a distinct glycosyltransferase fold. Nat Commun, 2014, 5: 4339

35 Albesa-Jové D, Giganti D, Jackson M, et al. Structure-function relationships of membrane-associated GT-B glycosyltransferases. Glycobiology, 2014, 24: 108-124

36 Vocadlo D J, Davies G J. Mechanistic insights into glycosidase chemistry. Curr Opin Chem Biol, 2008, 12: 539-555

37 Monegal A, Planas A. Chemical rescue of $\alpha 3$-galactosyltransferase. Implications in the mechanism of retaining glycosyltransferases. J Am Chem Soc, 2006, 128: 16030-16031

38 Gómez H, Polyak I, Thiel W, et al. Retaining glycosyltransferase mechanism studied by qM/MM methods: Lipopolysaccharyl- $\alpha-1,4-$ galactosyltransferase $\mathrm{C}$ transfers $\alpha$-galactose via an oxocarbenium ion-like transition state. J Am Chem Soc, 2012, 134: 4743-4752

39 Vetting M W, Frantom P A, Blanchard J S. Structural and enzymatic analysis of MshA from Corynebacterium glutamicum. J Biol Chem, 2008, 283: $15834-15844$

40 Frantom P A, Coward J K, Blanchard J S. UDP-(5F)-GlcNAc acts as a slow-binding inhibitor of MshA, a retaining glycosyltransferase. J Am Chem Soc, 2010, 132: 6626-6627

41 Lepak A, Gutmann A, Kulmer S T, et al. Creating a water-soluble resveratrol-based antioxidant by site-selective enzymatic glucosylation. Chembiochem, 2015, 16: 1870-1874

42 Takahashi K, Sun Y, Yanagiuchi I, et al. Stevioside enhances apoptosis induced by serum deprivation in PC12 cells. Toxicol Mech Methods, 2012, 22: 243-249

43 Geeraert B, Crombé F, Hulsmans M, et al. Stevioside inhibits atherosclerosis by improving insulin signaling and antioxidant defense in obese insulin-resistant mice. Int J Obes, 2010, 34: 569-577

44 Li Y, Baldauf S, Lim E K, et al. Phylogenetic analysis of the UDP-glycosyltransferase multigene family of Arabidopsis thaliana. J Biol Chem, 2001, 276: 4338-4343

45 Ross J, Li Y, Lim E K, et al. Higher plant glycosyltransferases. Genome Biol, 2001, 2: reviews3004.1

46 George Thompson A M, Iancu C V, Neet K E, et al. Differences in salicylic acid glucose conjugations by UGT74F1 and UGT74F2 from Arabidopsis thaliana. Sci Rep, 2017, 7: 46629

47 Modolo L V, Li L, Pan H, et al. Crystal structures of glycosyltransferase UGT78G1 reveal the molecular basis for glycosylation and deglycosylation of (iso)flavonoids. J Mol Biol, 2009, 392: 1292-1302

48 Hiromoto T, Honjo E, Noda N, et al. Structural basis for acceptor-substrate recognition of UDP-glucose: Anthocyanidin 3-O-glucosyltransferase from Clitoria ternatea. Protein Sci, 2015, 24: 395-407

49 Wetterhorn K M, Gabardi K, Michlmayr H, et al. Determinants and expansion of specificity in a trichothecene UDP-Glucosyltransferase from Oryza sativa. Biochemistry, 2017, 56: 6585-6596

50 Wetterhorn K M, Newmister S A, Caniza R K, et al. Crystal structure of Os79 (Os04g0206600) from Oryza sativa: A UDP-glucosyltransferase involved in the detoxification of deoxynivalenol. Biochemistry, 2016, 55: 6175-6186

51 Hsu T M, Welner D H, Russ Z N, et al. Employing a biochemical protecting group for a sustainable indigo dyeing strategy. Nat Chem Biol, 2018, 14: 256-261

52 Yang T, Zhang J, Ke D, et al. Hydrophobic recognition allows the glycosyltransferase UGT76G1 to catalyze its substrate in two orientations. Nat Commun, 2019, 10: 3214

53 Lee S G, Salomon E, Yu O, et al. Molecular basis for branched steviol glucoside biosynthesis. Proc Natl Acad Sci USA, 2019, 116: 13131-13136

54 Wang J H, Nao J F, Zhang M, et al. 20(s)-ginsenoside Rg3 promotes apoptosis in human ovarian cancer HO-8910 cells through PI3K/Akt and XIAP pathways. Tumor Biol, 2014, 35: 11985-11994

55 Chen F, Deng Z Y, Zhang B, et al. Esterification of ginsenoside Rh2 enhanced its cellular uptake and antitumor activity in human HepG2 cells. J Agric Food Chem, 2016, 64: 253-261

56 Chen T, Li B, Qiu Y, et al. Functional mechanism of ginsenosides on tumor growth and metastasis. Saudi J Biol Sci, 2018, 25: $917-922$

57 Liu T G, Huang Y, Cui D D, et al. Inhibitory effect of ginsenoside Rg3 combined with gemcitabine on angiogenesis and growth of lung cancer in 
mice. BMC Cancer, 2009, 9: 250

Wang P, Wei Y, Fan Y, et al. Production of bioactive ginsenosides Rh2 and Rg3 by metabolically engineered yeasts. Metab Eng, 2015, 29: 97105

59 Ahmadi M K, Fang L, Moscatello N, et al. E. coli metabolic engineering for gram scale production of a plant-based anti-inflammatory agent. Metab Eng, 2016, 38: 382-388

60 Bi H, Wang S, Zhou W, et al. Producing gram-scale unnatural rosavin analogues from glucose by engineered Escherichia coli. ACS Synth Biol, 2019, 8: 1931-1940

\title{
Research progress in understanding the structure, mechanism, and engineering of plant glycosyltransferases
}

\author{
LIU MeiZi ${ }^{1,2}$, WANG DanDan ${ }^{1,2}$, QIN Chao ${ }^{3}$, WANG XiaoQiang ${ }^{1,2}$ \& SHEN YueQuan ${ }^{1,4}$ \\ 1 State Key Laboratory of Medicinal Chemical Biology, Nankai University, Tianjin 300350, China; \\ 2 College of Pharmacy, Nankai University, Tianjin 300350, China; \\ 3 Department of Anesthesiology, Tianjin Medical University General Hospital, Tianjin 300052, China; \\ 4 College of Life Sciences, Nankai University, Tianjin 300071, China
}

Glycosyltransferases (GTs) are found in a wide variety of organisms, where they are involved in maintaining cell metabolism through glycosylation reactions. Glycosyltransferases transfer activated sugar from donor molecules to a variety of acceptors, this can alter the hydrophilic properties, stability, and chemical properties as well as subcellular location and biological activity of the acceptors. Many compounds that are modified by glycosylation are important sources of drug molecules. However, natural products contain only small amounts of glycosylated compounds, and it is very difficult to extract and purify these compounds. Furthermore, during the chemical synthesis of glycosylated compounds, the raw material reagents and generated by-products tend to cause environmental pollution, and it is not possible to achieve glycosylation at the particular acceptor sites. Recently, GTs have garnered interest among researchers. Therefore, here we review the current structural studies and biotechnological applications of plant GTs that would provide useful information for glycosylation engineering and bioactive glycoside production.

plant glycosyltransferases, glycosylation reactions, crystal structure, biotechnological application

doi: $10.1360 / \mathrm{SSV}-2019-0164$ 